\title{
Fine particulate air pollution, resuspended road dust and respiratory health among symptomatic children
}

\author{
P. Tiitanen*, K.L. Timonen*, J. J. Ruskanen**, A. Mirme ${ }^{+}$, J. Pekkanen*
}

\begin{abstract}
Fine particulate air pollution, resuspended road dust and respiratory health among symptomatic children. P. Tiittanen, K.L. Timonen, J. Ruuskanen, A. Mirme, J. Pekkanen. (C) ERS Journals Ltd 1999.

ABSTRACT: The short-term association of particulate air pollution with peak expiratory flow rate (PEF) and respiratory symptoms was examined.

Forty-nine children with chronic respiratory symptoms aged 8-13 yrs were followed daily for six weeks in spring, 1995, in Kuopio, Finland. Daily concentrations of particulate material with a $50 \%$ cut-off aerodynamic diameter $\leq \mathbf{1 0} \mu \mathrm{m}$ and $\leq \mathbf{2 . 5}$ $\mu \mathrm{m}$ (PM10 and PM2.5, respectively), black carbon, and the number concentrations of particles from 0.01-10 $\mu \mathrm{m}$ diameter were measured. During the study period, PM10 were mainly resuspended soil and street dust, and the concentration was estimated using aluminum content of PM10 samples.

No consistent effect of particles was found as the associations varied by lag. Of the lags examined, only 1-day lagged PM2.5 was statistically significantly associated with morning PEF ( $\beta=-1.06, \mathrm{SE}=\mathbf{0 . 5 2}$ (per interquartile increase in pollutant)). Evening PEF was significantly associated with the 1-day lagged number of particles in the size range 0.1-1.0 $\mu \mathrm{m}(\beta=-1.56, \mathrm{SE}=0.72)$. One-day lagged PM10, PM2.5-10, PM2.5 and resuspended PM10, and 4-day average of PM2.5 were significantly associated with increased risk of cough. Given the short duration of the study, separating the effects of different types of particles was difficult.

The present study demonstrates the highly variable size and number distribution and chemical composition of particles in Finland, and underlines the importance of measuring the size and chemical composition of particles to determine which types of particles are associated with health effects.
\end{abstract}

Eur Respir J 1999; 13: 266-273.

\begin{abstract}
*Unit of Environmental Epidemiology, Division of Environmental Health, National Public Health Institute, Kuopio, Finland. **Dept of Environmental Sciences, University of Kuopio, Kuopio, Finland. Institute of Environmental Physics, University of Tartu, Tartu, Estonia.
\end{abstract}

Correspondence: P. Tiittanen

Unit of Environmental Epidemiology Division of Environmental Health

National Public Health Institute

P.O. Box 95

FIN-70701 Kuopio

Finland

Fax: 35817201265

Keywords: Air pollution panel study

particles

peak expiratory flow

respiratory symptoms

Received: April 241998

Accepted after revision October 251998

Partly supported by U.S. EPA grant no. R825295-01-0.
Many studies have reported that daily changes in particulate air pollution are associated with a variety of adverse respiratory outcomes, like symptoms, decline in lung function, hospital emergency admission and mortality $[1,2]$. More recently, similar associations have also been reported with cardiovascular outcomes, like hospital admissions and mortality $[3,4]$.

Most of the previous studies have reported associations between respiratory symptoms and particulate air pollution measured as particulate matter with a $50 \%$ cut-off aerodynamic diameter $10 \mu \mathrm{m}$ (PM10) in winter, when particles originate mainly from combustion processes [2]. There are only a few studies on the health effects of particulate air pollution originating from soil [5-9]. They suggest that PM10 from soil may also have adverse health effects on the respiratory system, but the effect may be smaller than for PM10 from combustion processes.

Particles in air tend to follow a trimodal distribution. Particles $<2.5 \mu \mathrm{m}$ in diameter are generally called "fine", and those $>2.5 \mu \mathrm{m}$ in diameter as "coarse". Fine particles have traditionally been divided into two modes: the nuclei mode (ultrafine particles) and the accumulation mode. The large number of ultrafine particles has been suggested as a possible mechanism for the health effects of PM10 [10].
However, few studies have been published on the health effects of ultrafine particles. The number concentration of ultrafine particles has been found to be associated with decreased lung function and increased respiratory symptoms at least as strongly as the mass of coarse particles [11-13]. Toxicological studies have shown that ultrafine titanium [14] and teflon $[15,16]$ particles produce acute pulmonary toxicity and inflammatory reactions in rats.

The present study investigated the association of particulate air pollution with peak expiratory flow rate (PEF) and respiratory symptoms in spring, during which the major source of PM10 particles in Finnish cities is soil and street dust [17]. During the winter, the use of studded tyres produces asphalt dust. This dust together with street sanding and particles from combustion processes are deposited on the icy street surfaces. When the snow melts from the streets, a proportion of this dust is resuspended into the air by traffic and wind. This causes dust episodes with high particle concentrations, during which PM10 levels exceed the national air quality guideline $\left(70 \mu \mathrm{g} \cdot \mathrm{m}^{-3}, 24-\right.$ $\mathrm{h}$ mean) in most Finnish cities. In addition to PM10, we also examined the association of particles of different sizes between 0.01 and $10 \mu \mathrm{m}$ with PEF and respiratory symptoms. 


\section{Subjects and methods}

\section{Study location}

The study was conducted in Kuopio, a town of 85,000 inhabitants in eastern Finland. The main sources of particulate air pollution in Kuopio are traffic, a peat-fired power plant connected to a municipal district heating system, and a corrugated cardboard mill. More than 90\% of the buildings in Kuopio are heated from the municipal district heating system. In normal use, the peat-fired power produces all the necessary heating energy.

\section{Study design and subjects}

A total of 76 children aged 8-13 yrs from four primary schools in the centre of the town, who had in the previous year, participated in the Pollution Effects on Asthmatic Children in Europe (PEACE) study $[18,19]$ were invited to the study. Second invitation letters were sent to those who did not respond to the first letter. Fifty-eight symptomatic children agreed to take part and started the follow-up study. The child was defined as symptomatic if she/he had reported at least one of the following chronic respiratory symptoms in the PEACE screening questionnaire: "wheeze in the past 12 months", "attacks of shortness of breath with wheezing in the past 12 months", "dry cough during night apart from colds in the past 12 months" or "ever doctor-diagnosed asthma" [18]. All the included children had been characterized with skin prick tests and spirometry in the PEACE study [18]. Of the included children, $15(26 \%)$ had doctor-diagnosed asthma, 15 (26\%) were using medication for asthma, and 37 (64\%) were atopic. In addition, nonsymptomatic children $(n=32)$ who had previously taken part in a asthma prevalence study [20] based on the PEACE study population, were also invited, but due to the small number of children participating in the present study $(n=13)$, they were not included in the present analyses.

The children were followed-up for six weeks, from March 13 to April 23, 1995. The first week was excluded from the analyses as a learning period. In addition, the days spent out of town were excluded. The diary study followed the PEACE study protocol [21]. The children measured their PEF three times every morning and every evening in the standing position with a mini-Wright Peak Flow meter (Airmed, Clement Clarke International Ltd., Essex, UK) before taking any respiratory medication. All three PEF readings were noted in a diary, and the greatest of these three readings was used in the analyses.

The children also kept a daily diary of respiratory symptoms with the help of their parents. The following symptoms were reported on a scale of $0-2(0=$ no symptom, $1=$ slight, $2=$ moderate/severe): cough, phlegm, runny or stuffed nose, awakened with breathing problems, shortness of breath, wheeze, attack(s) of shortness of breath with wheeze, fever, eye irritation and sore throat. For symptom analyses, four binary variables indicating daily symptoms were created. The child was defined as having a symptom if a slight or moderate/severe symptom was recorded on that day. Lower respiratory symptoms (LRS) were defined as being present when the child reported on a given day having at least one of the following symptoms: shortness of breath, wheeze, or attacks of shortness of breath. Upper respiratory symptoms (URS) were defined as being present when the child reported having a sore throat, or runny or stuffed nose. Cough and phlegm symptom recordings were dichotomized before the analyses.

The children were also asked to report in the diary the name of the medicine and the amount taken on that day. Two binary variables indicating medication use were created for the analyses: use of bronchodilator (salbutamol, terbutaline) and use of preventive medication (inhaled corticosteroids, sodium cromoglycate, nedocromil).

Only those symptomatic children who had completed the diary on $>21(60 \%)$ days during the follow-up were considered for the analyses $(n=50)$. Because the PEF measurements of one child fluctuated unreliably, only 49 children (27 female and 22 male, mean ( \pm SD) age $10 \pm 1.6$ yrs) were included in the analyses. Among these 49 children the mean number of days with nonmissing symptom or PEF data per child were 29 and 28, respectively.

The children also completed a questionnaire on respiratory symptoms during the study. This was the same questionnaire as in the screening phase of the PEACE study [18]. Of the 47 children who returned the questionnaire, 13 (28\%) had doctor-diagnosed asthma. During the last 12 months, $10(21 \%)$ had suffered from shortness of breath and $18(38 \%)$ from dry cough, and six (13\%) had wheezed. Eleven $(23 \%)$ were exposed to passive smoking at home. Twenty-four (49\%) children had positive skinprick reactions to birch, common alder, timothy or mugwort pollen.

The study protocol was approved by the Ethical Committee of the University of Kuopio and of the Kuopio University Hospital. Written consent was obtained from both the children and their parents.

\section{Air quality measurements}

All air quality measurements (except ozone) were carried out at the same site in the centre of Kuopio, at least 50 $\mathrm{m}$ from any of the surrounding streets. Particle size distributions were measured with an electric aerosol spectrometer (EAS), which is based on the electrical charging and the analysis of electrical mobility of aerosol particles [22]. EAS enables analysis of particle sizes from $0.01-10 \mu \mathrm{m}$ in 12 consecutive size classes every $15 \mathrm{~min}$. For the analyses, the 12 size classes were combined into three size classes by counting together particle numbers $(\mathrm{PN})$ in four adjacent classes. The cut-off points of upper diameters were $0.1 \mu \mathrm{m}$ (PN0.01-0.1), $1.0 \mu \mathrm{m}$ (PNo.1-1.0) and $10 \mu \mathrm{m}$ (PN1.0-10). This division broadly reflects the trimodal distribution of particulate air pollution: ultrafine particles, particles in accumulation mode and coarse particles. Only PN0.01-0.1 and PN0.1-1.0 were used in the present analyses as the small number of large particles in air is more accurately reflected in mass-based measurement (i.e. PM10).

PM10 and particulate material with a 50\% cut-off aerodynamic diameter $\leq 2.5 \mu \mathrm{m}$ (PM2.5) were collected with single stage Harvard impactors [23]. PM10 was also measured continuously with a TEOM 1400A ambient particulate monitor (Rupprecht \& Patashnick Co., Albany, NY, USA). The difference between PM10 and PM2.5 was calculated and used as an indicator for coarse particles 
(PM2.5-10). Total suspended particles (TSP) were collected with a high-volume sampler using the SFS 3863 method, which is based on US Environmental Protection Agency standard (NAAQS 40 CFR 50). Concentration of black carbon (BC) was measured with a computer-controlled aethalometer (Magee AE-9 Aethalometer $\mathbb{R}$; Magee Scientific Co., Berkeley, CA, USA) [24]. Gaseous pollutants were measured with continuously recording monitors: nitric oxide and nitrogen dioxide, with a chemiluminescence method (Monitor Labs 8840; Monitor Labs Inc., San Diego, CA, USA), sulphur dioxide with ultra-violet fluorescence meth-od (Monitor Labs 8850), and carbon monoxide with a nondispersive infrared method (Thermo Environmental 48; Franklin, MA, USA). Concentrations of ozone were measured with an ultra-violet photometric ozone analyser (Dasibi 1008-RS; Dasibi Environmental Co., Glendale, CA, USA) at the measurement site, which is $3.5 \mathrm{~km}$ west of the centre of Kuopio.

Meteorological data (wind speed, wind direction and temperature) were obtained from the municipal weather station network, and relative humidity was measured at Kuopio airport. Daily pollen counts collected with a Burkard volumetric pollen trap (Burkard Manufacturing Co., Hertfordshire, UK) were obtained from the Finnish Aerobiology Group [25]. The sampler was located at the University of Kuopio, $3 \mathrm{~km}$ west of the centre of Kuopio. Filters for PM10 samples were always changed at approximately 13:00 $\mathrm{h}$. Twenty-four-h means were also calculated for the other pollutants and meteorological parameters from 13:00 h to $13: 00 \mathrm{~h}$.

The elemental composition of PM10 samples was analysed using the inductively coupled plasma-mass spectrometry (ICP-MS) technique [17]. The sources of PM10 were examined with receptor modelling based on principal component analysis and multiple linear regression. The aluminium content of the PM10 filters was used as a marker for PM10 particles originating from soil, for two reasons. Firstly, principal component analysis of chemical elements in PM10 revealed that the first factor was clearly related to soil particles, and aluminium was strongly loaded only in this factor. Secondly, we have previously found that aluminium is a good marker for PM10 originating from soil [17]. The daily concentration of PM10 was regressed against the daily aluminium content of filters $\left(r^{2}=0.96\right)$. Resuspended PM10 was estimated by multiplying the daily content of aluminium with the regression coefficient of aluminium ( $\beta=15.37)$ obtained from the regression model. As a sensitivity analysis, $\mathrm{CO}$, which is a marker for traffic [17], was included in the regression model, as well as aluminium, to estimate resuspended PM10. However, the estimated concentration of resuspended PM10 was practically unchanged.

\section{Statistical analysis}

Spearman's rank correlations between the air pollutants and minimum temperature were calculated. Adjusted daily prevalences of respiratory symptoms and medication use, and adjusted daily average PEF deviations in the tertiles of different particulate air pollutants were calculated as least squares means with the GLM procedure [26].
The association of particulate air pollution (lags 0-3 and 4-day average as an average of lags 0-3) with PEF was analysed by a linear multiple regression model. The daily deviation from each child's mean morning and evening PEF was used as a dependent variable, and the analyses were carried out separately for morning and evening PEF. Lag 0 for morning PEF was defined as the 24-h period from yesterday noon to the present day noon, and that for evening PEF from present day noon to the next day noon. The model was estimated by the MIXED procedure [27], and first-order autoregressive covariance structure for errors was used to account for the dependence of the repeated measurements on the same child.

The basic model included several confounders, which were selected based on both previous knowledge and on their influence on the estimate of the regression coefficient for particulate air pollutants. Day of study and day of study squared were included in the regression model to eliminate the time trend, and a dummy variable for the weekend was introduced to control for the weekend effect. Daily minimum temperature, daily average relative humidity and a dummy variable for daily total pollen count were also included in the model. Based on the allergy thresholds values in use in Nordic countries, pollen count (mostly alder pollen) was divided into two categories using 100 grains $\cdot \mathrm{m}^{-3}$ as a cut-off point [28]. Possible nonlinear association of PEF with particulate air pollution and confounders was checked using generalized additive models in S-PLUS [29]. Locally weighted least-squares regression was used as a smooth function. However, the linearity assumption was an adequate description of the associations explored.

As a sensitivity analysis, the association between particulate air pollution and PEF was also analysed using aggregated data. The daily deviations from each child's mean morning and evening PEF were first calculated and then averaged to obtain the mean daily deviation $(\triangle \mathrm{PEF})$, separately for morning and evening PEF. The association of particulate air pollution (lags $0-3$ and 4-day average) with $\triangle \mathrm{PEF}$ was examined by a linear first-order autoregressive model controlling for day of study, day of study squared, weekend, minimum temperature, relative humidity and pollen. The model was estimated with the AUTOREG procedure [30].

The association of cough, phlegm, URS, LRS and use of medication (bronchodilator and preventive medication, separately) with particulate air pollution was investigated using the random effects logistic regression model. The model was estimated with the SAS macro GLIMMIX [31]. There was only a random intercept in the model, and no other random effects were specified. The simple random effects model assumes independent errors. However, firstorder autoregressive covariance structure for errors was more appropriate in this model and it was used. Confounders were selected from the analyses of respiratory symptoms and medication use, as in the analysis of PEF, and the same confounders were used. The analyses were also repeated, first aggregating the data into daily prevalences of respiratory symptoms and use of medication. Daily prevalences were then used as dependent variables in multiple logistic regression models. The conclusions from these results were identical to those from the results presented. 


\section{Results}

Particulate air pollution levels were generally low (table 1) except for a few days (figs. 1 and 2). The intercorrelations between TSP, PM10, PM2.5-10, PM2.5, and resuspended PM10 were high (table 2), and most indicators of particulate air pollution peaked on the same days. However, at the beginning of the period there was a peak in BC and smaller particles, while PM10 showed only a small peak. During this peak there was a strong atmospheric inversion above the city and therefore particles probably came mostly from combustion processes. The remaining peaks in PM10 were mainly resuspended dust. The levels of the concentrations of the other measured air pollutants were low, and did not exceed the national air quality guidelines (table 1). The intercorrelations between indicators of particulate air pollution and $\mathrm{CO}$ were high (table 2). In addition, $\mathrm{NO}_{2}$ and $\mathrm{O}_{3}$ were highly correlated with PN0.01-0.1 and BC.

There was more cough and LRS in the two highest tertiles of fine particles (table 3 ). Bronchodilator use seemed to increase over the tertiles of fine and ultrafine particles. However, the results for bronchodilator and preventive medication use, and for LRS suffer from a low number of cases. Also, most of the cases were children who had LRS or used medication every day. The increase in fine particles was associated with a decrease in morning PEF, whereas evening PEF decreased along the tertiles of both fine and ultrafine particles.

The results of the multiple linear regressions suggest that increases in the concentrations of particulate air pollutants for lag 1 were associated with a decrease in the morning PEF (table 4). Although both fine and coarse particles had a negative effect on PEF, only the estimate for PM2.5 reached statistical significance $(\mathrm{p}<0.05)$. However, the estimates change their sign considerably from lag to lag. Evening PEF was also negatively associated with all indicators of particulate air pollutants at lag 1 (table 4). Fine particles, espec-ially particles in the accumulation mode, tended to have a stronger effect on evening PEF than coarse particles. However, only the estimate for PN0.1-1.0

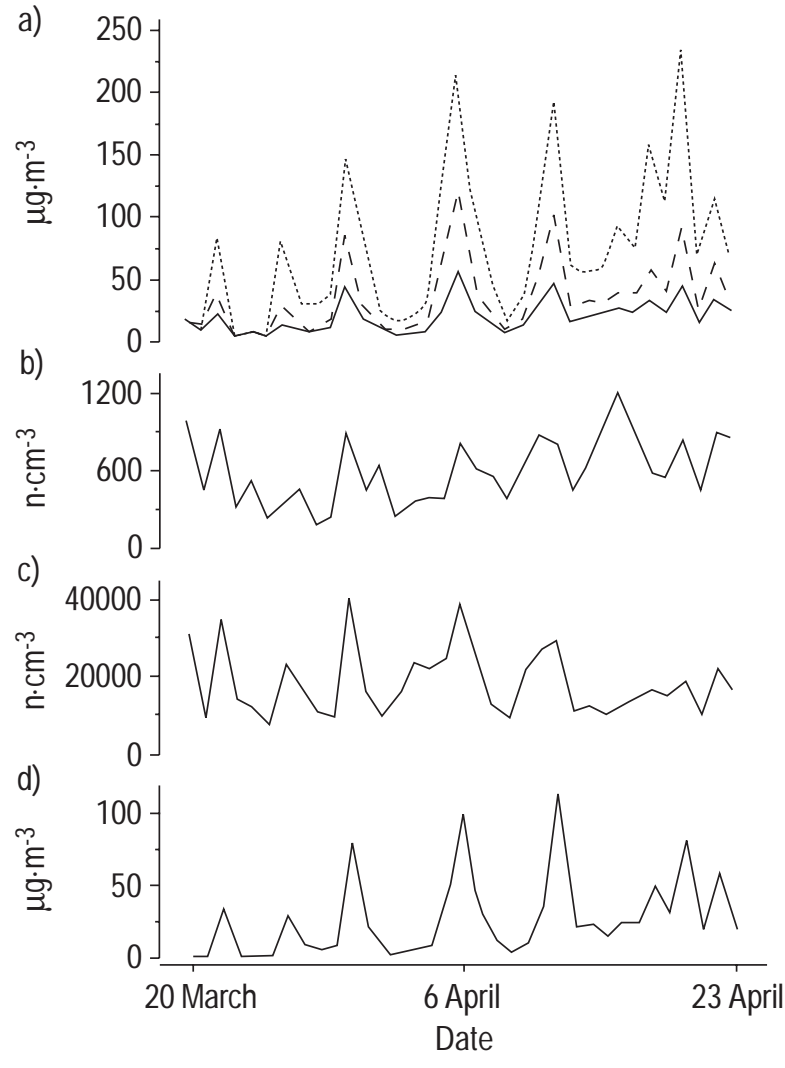

Fig. 1. - Daily average levels of a) total suspended particles (TSP; - - - ), particulate material with a $50 \%$ cut-off aerodynamic diameter $\leq 10 \mu \mathrm{m}\left(\mathrm{PM} 10 ;-{ }_{-}\right)$and $\leq 2.5 \mu \mathrm{m}(\mathrm{PM} 2.5 ;-1-)$; b ) number of particles in size range $0.1-1.0 \mu \mathrm{m}$ (PNo.1-1.0) and c) $0.01-0.1 \mu \mathrm{m}$ (PNo.01-0.1); and d) estimated resuspended PM10.

was statistically significant. The results of the aggregated data analysis were similar to the results in table 4, except that at lag 1 PM10 was also statistically significantly associated with morning PEF (results not shown).

To check the sensitivity of the results on the selected confounders, the association between PN0.1-1.0 and evening

Table 1. - Daily average levels of particulate and gaseous air pollutants and minimum temperature during the study period $(n=35$, except for carbon monoxide, sulphur dioxide and nitrogen dioxide $(n=34)$

\begin{tabular}{|c|c|c|c|c|c|}
\hline & \multicolumn{5}{|c|}{ Percentile } \\
\hline & Min. & $25 \%$ & $50 \%$ & $75 \%$ & Max. \\
\hline $\mathrm{TSP} \mu \mathrm{g} \cdot \mathrm{m}^{-3}$ & 6 & 25 & 57 & 103 & 234 \\
\hline $\mathrm{PM} 10 \mu \mathrm{g} \cdot \mathrm{m}^{-3}$ & 5 & 12 & 28 & 43 & 122 \\
\hline PM2.5-10 $\mu \mathrm{g} \cdot \mathrm{m}^{-3}$ & 0.2 & 3 & 8 & 20 & 67 \\
\hline PM2.5 $\mu \mathrm{g} \cdot \mathrm{m}^{-3}$ & 3 & 9 & 15 & 23 & 55 \\
\hline PNo.1-1.0 $\mathrm{n} \cdot \mathrm{cm}^{-3}$ & 183 & 366 & 538 & 820 & 1190 \\
\hline PN0.01-0.1 $\mathrm{n} \cdot \mathrm{cm}^{-3}$ & 6980 & 10200 & 14700 & 22700 & 40200 \\
\hline Res. PM10 $\mu \mathrm{g} \cdot \mathrm{m}^{-3}$ & 0.5 & 3 & 17 & 33 & 112 \\
\hline Black carbon $\mathrm{ng} \cdot \mathrm{m}^{-3}$ & 291 & 576 & 788 & 1120 & 2120 \\
\hline $\mathrm{CO} \mathrm{mg} \cdot \mathrm{m}^{-3}$ & 0.1 & 0.3 & 0.4 & 0.5 & 1.0 \\
\hline $\mathrm{SO}_{2} \mu \mathrm{g} \cdot \mathrm{m}^{-3}$ & 0 & 0.1 & 0.7 & 2.6 & 5.2 \\
\hline $\mathrm{NO}_{2} \quad \mu \mathrm{g} \cdot \mathrm{m}^{-3}$ & 5 & 10 & 15 & 23 & 46 \\
\hline $\mathrm{O}_{3} \mu \mathrm{g} \cdot \mathrm{m}^{-3}$ & 0 & 28 & 35 & 38 & 50 \\
\hline Min. temp. ${ }^{\circ} \mathrm{C}$ & -13 & -6 & -2 & 1 & 5 \\
\hline
\end{tabular}

TSP: total suspended particulates; PM10: particulate matter with a 50\% cut-off aerodynamic diameter $\leq 10 \mu$ m; PM2.5: particulate matter with a $50 \%$ cut-off aerodynamic diameter $\leq 2.5 \mu \mathrm{m}$; PM2.5-10: difference between PM10 and PM2.5; PN0.1-1.0: number concentration of particles in the size range $0.1-1.0 \mu \mathrm{m}$; PN0.01-0.1: number concentration of particles in size range $0.01-0.1 \mu \mathrm{m}$; Res. PM10: estimated resuspended PM10; Min temp.: daily minimum temperature; Min: minimum; Max: maximum. 

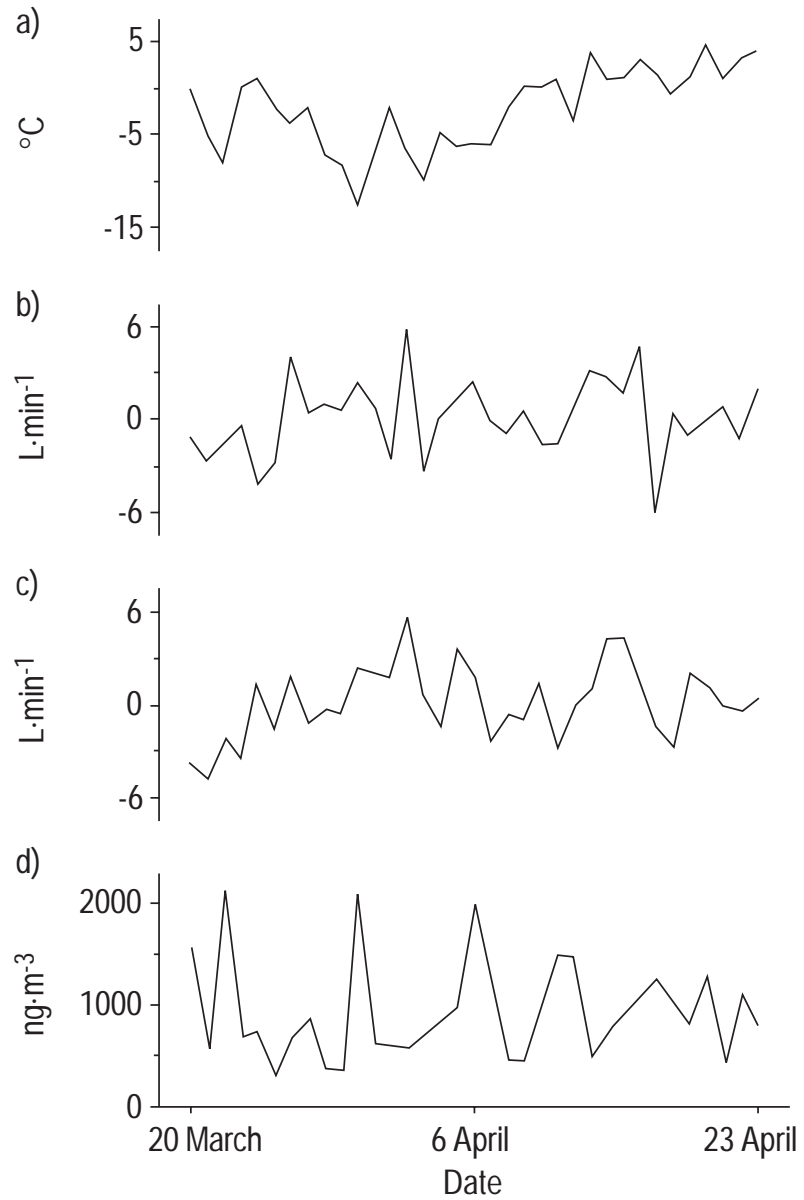

Fig. 2. - a) Daily minimum temperature $\left({ }^{\circ} \mathrm{C}\right)$, b) average morning and c) evening peak expiratory flow (PEF) deviations, and d) average levels of black carbon.

PEF, as well as PM2.5 and morning PEF, was examined more thoroughly. The crude regression coefficient for PN $0.1-1.0$ was -1.53 and for PM2.5 -0.26. Adding confounders strengthened the association of PM2.5 with morning PEF, but had little effect on the coefficient, for PN0.1-1.0. Including indicator variables for the Easter holiday, the use of bronchodilators, preventive medication or exposure to tobacco smoking at home in the regression model did not change the results. Replacing 1-day lagged values of minimum temperature, relative humidity and pollen with the same day values in the regression models for evening PEF did not alter the results.

The results of two pollutant models, i.e. having an indicator for particulate mass concentration (PM2.5 or PM10) and an indicator for particle number concentration (PNo.010.1 or PN0.1-1.0) in the same model, were the following. The association between morning PEF and 1-day lagged PM2.5 decreased when PN0.01-0.1 was in the same model ( $p=0.09$ for PM2.5). Also, all the other associations between morning PEF and 1-day lagged particulates decreased $(p>0.10)$. The sign of the regression coefficients still varied by lag. The association between evening PEF and 1-day lagged PN0.1-1.0 was still statistically significant when PM10 (for PN0.1-1.0, $\beta=-2.18, \mathrm{SE}=0.93$ ) or PM2.5 (for PN0.1-1.0, $\beta=-2.43, \mathrm{SE}=1.06)$ was included in the model. Lag 2 for PN0.1-1.0 was still positive, while other lags were negative. Four-day average of PN0.1-1.0 $(\beta=-5.15, \mathrm{SE}=2.48)$ also reached statistical significance when PM2.5 was included in the model.

Adding gaseous pollutants one at a time in the model, i.e. having both a particulate and a gaseous pollutant in the model at the same time, changed the results for lag 1 as follows. In the case of morning $\mathrm{PEF}, \mathrm{O}_{3}$ strengthened the observed associations and all indicators of particulate air pollution reached statistical significance. Introducing either $\mathrm{NO}_{2}$ or $\mathrm{SO}_{2}$ in the model did not change the results markedly. In the analysis of evening PEF, $\mathrm{SO}_{2}$ did not alter the results. There were no statistically significant associations remaining between particulate air pollutants and evening PEF when $\mathrm{NO}_{2}$ or $\mathrm{O}_{3}$ was included in the model. However, $\mathrm{NO}_{2}$ and $\mathrm{O}_{3}$ were highly correlated with $\mathrm{PN} 0.01-$ 0.1 , thus causing multi-colinearity in the model. For the same reason, CO was not used at all in two-pollutant models. None of the gaseous pollutants at any lag, except the 4-day average of $\mathrm{SO}_{2}$ and the 4-day average of $\mathrm{O}_{3}$, were statistically significantly associated either with morning or evening PEF, when a gaseous pollutant was the only pollutant in the model.

Cough had a positive, statistically significant association with PM10, PM2.5-10, PM2.5, and resuspended PM10 at lag 2 and with 4-day average PM2.5 (table 5). An increase of $31 \mu \mathrm{g} \cdot \mathrm{m}^{-3}$ in PM10 at lag 2 resulted in a $15 \%$ increase in cough, and an increase of $14 \mu \mathrm{g} \cdot \mathrm{m}^{-3}$ in the 4-day

Table 2. - Spearman's correlations* between daily average levels of particulate and gaseous air pollutants and minimum temperature during the study period $(n=35$, except in pairs containing carbon monoxide, sulphur dioxide or nitrogen dioxide $\mathrm{n}=34$ )

\begin{tabular}{|c|c|c|c|c|c|c|c|c|c|c|c|c|}
\hline & PM10 & PM2.5-10 & PM2.5 & PN0.1-1.0 & PN0.01-0.1 & Res. PM10 & Black carbon & $\mathrm{CO}$ & $\mathrm{SO}_{2}$ & $\mathrm{NO}_{2}$ & $\mathrm{O}_{3}$ & Min. temp. \\
\hline TSP & 0.98 & 0.97 & 0.92 & 0.50 & 0.54 & 0.97 & 0.63 & 0.53 & 0.27 & 0.32 & -0.04 & 0.09 \\
\hline PM10 & & 0.97 & 0.97 & 0.62 & 0.61 & 0.96 & 0.74 & 0.56 & 0.28 & 0.42 & -0.14 & 0.12 \\
\hline PM2.5-10 & & & 0.90 & 0.47 & 0.63 & 0.98 & 0.67 & 0.54 & 0.29 & 0.43 & -0.13 & 0.03 \\
\hline PM2.5 & & & & 0.75 & 0.56 & 0.89 & 0.77 & 0.56 & 0.32 & 0.41 & -0.12 & 0.20 \\
\hline $\mathrm{PN} 0.1-1.0$ & & & & & 0.39 & 0.45 & 0.79 & 0.50 & 0.27 & 0.30 & -0.14 & 0.41 \\
\hline PN0.01-0.1 & & & & & & 0.56 & 0.81 & 0.65 & 0.14 & 0.79 & -0.69 & -0.29 \\
\hline Res. PM10 & & & & & & & 0.62 & 0.51 & 0.25 & 0.36 & -0.10 & 0.09 \\
\hline Black carbon & & & & & & & & 0.69 & 0.24 & 0.63 & -0.52 & 0.05 \\
\hline $\mathrm{CO}$ & & & & & & & & & 0.10 & 0.38 & -0.33 & -0.14 \\
\hline $\mathrm{SO}_{2}$ & & & & & & & & & & 0.25 & 0.21 & -0.04 \\
\hline $\mathrm{NO}_{2}$ & & & & & & & & & & & -0.61 & -0.15 \\
\hline $\mathrm{O}_{3}$ & & & & & & & & & & & & 0.30 \\
\hline
\end{tabular}

*: all correlations above 0.35 or under -0.35 are significant at $\mathrm{p}<0.05$. For definitions see footnote to table 1 . 
Table 3. - Adjusted ${ }^{*}$ daily prevalences of respiratory symptoms and medication use, and the adjusted daily average peak expiratory flow (PEF) deviations $\left(\mathrm{L} \cdot \mathrm{min}^{-1}\right)$ in the tertiles of particulate air pollutants at lag 1

\begin{tabular}{lccccccccccccccc}
\hline & Range & $\begin{array}{c}\text { No of } \\
\text { days }\end{array}$ & $\begin{array}{c}\text { Preventive } \\
\text { medication }\end{array}$ & $\begin{array}{c}\text { Bronchodilator } \\
\text { medication }\end{array}$ & Cough & Phlegm & URS & LRS & Morning & Evening \\
\hline PM2.5 & -8.9 & 11 & 0.15 & 0.03 & 0.32 & 0.18 & 0.51 & 0.02 & 1.63 & 2.02 \\
& $9-20.2$ & 12 & 0.16 & 0.03 & 0.36 & 0.20 & 0.52 & 0.04 & 1.17 & 0.72 \\
& $20.2-$ & 12 & 0.16 & 0.05 & 0.37 & 0.19 & 0.52 & 0.04 & -1.11 & 0.28 \\
PN0.01-0.1 & -11300 & 11 & 0.15 & 0.03 & 0.35 & 0.19 & 0.50 & 0.03 & 0.46 & 1.55 \\
& $11300-20500$ & 12 & 0.16 & 0.04 & 0.35 & 0.19 & 0.52 & 0.04 & 0.90 & 0.49 \\
& $20500-$ & 12 & 0.16 & 0.05 & 0.35 & 0.20 & 0.55 & 0.04 & -0.01 & 0.22 \\
Res. PM10 & -5 & 11 & 0.16 & 0.03 & 0.34 & 0.20 & 0.52 & 0.03 & 0.86 & 1.64 \\
& $5-22$ & 12 & 0.15 & 0.04 & 0.35 & 0.19 & 0.51 & 0.03 & 1.16 & 0.53 \\
& $22-$ & 12 & 0.16 & 0.04 & 0.36 & 0.19 & 0.53 & 0.04 & -0.39 & 0.75 \\
\hline
\end{tabular}

*: evening PEF, symptoms and medication use adjusted for day, day ${ }^{2}$, weekend, minimum temperature (lag 1), relative humidity (lag 1 ) and pollen (lag 1), and morning PEF adjusted for day, day ${ }^{2}$, weekend, minimum temperature (lag 0 ), relative humidity (lag 0 ) and pollen (lag 0). URS: upper respiratory symptom; LRS: lower respiratory symptom. For remaining definitions see footnote to table 1.

average PM2.5 increased cough by $48 \%$. Adding gaseous pollutants one at a time in the model did not change the results for cough.

Phlegm had a positive, but not statistically significant association with PM10, PM2.5-10, PM2.5, and resuspended PM10 for lag 2. Particulate air pollutants at any lag did not seem to affect URS. Random effects models for LRS and use of medication (bronchodilator and preventive medication, separately) did not converge, possibly due to the low number of cases.

\section{Discussion}

The concentrations of PM10 exceeded the national air quality guidelines of $70 \mu \mathrm{g} \cdot \mathrm{m}^{-3}$ for $24-\mathrm{h}$ mean in four days, but the levels of particulate air pollutants were generally low during the study period. The contribution of resuspended particles to PM10 was large during the study period.
On the first days of the study period there was a clear peak in ultrafine particles (PN0.01-0.1), but no peak in the more coarse particulate matter. However, the concentration of $\mathrm{BC}$ also peaked indicating that the source was traffic. During the middle part of the study period, the peaks in TSP, PM10, and PM2.5 coincided with the peaks in PN0.010.1 . Finally, during the last 10 days of the study period the concentrations of TSP and PM10 were disproportionally elevated compared to the concentrations of PN0.01-0.1. This may be due to the fact that in the middle part of the study period the resuspended dust also contained fine particles, which are deposited on the snowy and icy streets during the winter. Later the resuspended dust contained mainly coarse particles (e.g. street sand).

The exact mechanisms for the health effects of particulates are still unknown. Based on animal studies and theoretical calculations, a potential explanation is the very high number of ultrafine particles in the urban air [10]. It has been shown that fumes from the thermodegradation of Teflon produce acute inflammation, haemorrhage, oedema

Table 4. - Adjusted associations of daily average levels of particulate air pollutants with morning* and evening ${ }^{\dagger}$ PEF deviations $\left(L \cdot \mathrm{min}^{-1}\right)$

\begin{tabular}{|c|c|c|c|c|c|c|c|c|c|c|}
\hline \multirow[b]{2}{*}{ Morning PEF } & \multicolumn{2}{|c|}{ Lag 0} & \multicolumn{2}{|c|}{ Lag 1} & \multicolumn{2}{|c|}{ Lag 2} & \multicolumn{2}{|c|}{ Lag 3} & \multicolumn{2}{|c|}{ 4-day average } \\
\hline & & & & & & & & & & \\
\hline $\mathrm{n}$ & \multicolumn{2}{|c|}{1353} & \multicolumn{2}{|c|}{1307} & \multicolumn{2}{|c|}{1260} & \multicolumn{2}{|c|}{1213} & \multicolumn{2}{|c|}{1213} \\
\hline PM10 & 0.74 & $(0.51)$ & -1.01 & $(0.53)^{\S}$ & 0.46 & $(0.59)$ & -0.53 & $(0.52)$ & -0.77 & $(1.46)$ \\
\hline PM2.5-10 & 0.78 & $(0.49)$ & -0.86 & $(0.49)^{\S}$ & 0.35 & $(0.56)$ & -0.39 & $(0.47)$ & -0.46 & (1.34) \\
\hline PM2.5 & 0.62 & $(0.50)$ & -1.06 & $(0.52)$ & 0.54 & $(0.57)$ & -0.70 & $(0.54)$ & -1.08 & $(1.45)$ \\
\hline PNo.1-1.0 & 0.19 & $(0.71)$ & -1.24 & $(0.72)^{\S}$ & 0.82 & $(0.76)$ & -0.07 & $(0.97)$ & -0.75 & (2.34) \\
\hline PNo.01-0.1 & 0.20 & $(0.59)$ & -0.68 & $(0.56)$ & 0.88 & $(0.61)$ & -0.46 & $(0.55)$ & -0.43 & $(1.80)$ \\
\hline Res. PM10 & 0.84 & $(0.50)^{\S}$ & -0.85 & $(0.51)^{\S}$ & 0.47 & $(0.56)$ & -0.46 & $(0.49)$ & -0.18 & (1.53) \\
\hline Black carbon & 0.28 & $(0.42)$ & -0.74 & $(0.42)^{\S}$ & 0.69 & $(0.46)$ & -0.19 & $(0.44)$ & -0.18 & (1.38) \\
\hline \multicolumn{11}{|l|}{ Evening PEF } \\
\hline $\mathrm{n}$ & \multicolumn{2}{|c|}{1362} & \multicolumn{2}{|c|}{1362} & \multicolumn{2}{|c|}{1315} & \multicolumn{2}{|c|}{1268} & \multicolumn{2}{|c|}{1268} \\
\hline PM10 & 0.44 & $(0.42)$ & -0.33 & $(0.54)$ & 0.05 & $(0.53)$ & 0.27 & $(0.59)$ & 1.42 & $(1.54)$ \\
\hline PM2.5-10 & 0.45 & $(0.39)$ & -0.21 & $(0.51)$ & 0.02 & $(0.49)$ & 0.33 & $(0.56)$ & 1.61 & (1.38) \\
\hline PM2.5 & 0.39 & $(0.43)$ & -0.43 & $(0.52)$ & 0.08 & $(0.53)$ & 0.15 & $(0.57)$ & 0.85 & $(1.55)$ \\
\hline PNo.1-1.0 & 0.38 & $(0.73)$ & -1.56 & $(0.72)^{*}$ & 0.28 & $(0.73)$ & -0.50 & $(0.76)$ & -3.07 & (2.09) \\
\hline PNo.01-0.1 & 0.69 & $(0.53)$ & -1.00 & $(0.60)^{\S}$ & 0.49 & $(0.57)$ & -0.01 & $(0.62)$ & 1.05 & (1.88) \\
\hline Res. PM10 & 0.37 & $(0.40)$ & -0.11 & $(0.51)$ & 0.10 & $(0.51)$ & 0.42 & $(0.56)$ & 2.13 & (1.53) \\
\hline Black carbon & 0.35 & $(0.39)$ & -0.67 & $(0.43)$ & 0.28 & $(0.43)$ & -0.04 & $(0.47)$ & -0.38 & (1.39) \\
\hline
\end{tabular}

The data are presented as the regression coefficient $(\beta)$ and standard error (sE; in parenthesis) which are multiplied by the interquartile range of the pollutant. ${ }^{*}$ : adjusted for day, day ${ }^{2}$, weekend, minimum temperature (lag 0 ), pollen (lag 0 ) and first-order autocorrelation; ${ }^{\dagger}$ : adjusted for day, day ${ }^{2}$, weekend, minimum temperature (lag 1), relative humidity (lag 1), pollen (lag 1) and first-order autocorrelation; $\mathrm{p}<0.05 ;{ }^{\S}: \mathrm{p}<0.10$. For definitions see footnote to table 1 . 
Table 5. - Adjusted* associations of daily average levels of particulate air pollutants and cough

\begin{tabular}{lccccc}
\hline & $\begin{array}{c}\text { Lag 0 } \\
(\mathrm{n}=1441)\end{array}$ & $\begin{array}{c}\text { Lag 1 } \\
(\mathrm{n}=1441)\end{array}$ & $\begin{array}{c}\text { Lag 2 } \\
(\mathrm{n}=1393)\end{array}$ & $\begin{array}{c}\text { Lag 3 } \\
(\mathrm{n}=1345)\end{array}$ & $\begin{array}{c}\text { 4-day average } \\
(\mathrm{n}=1345)\end{array}$ \\
\hline OR 95\% CI & OR 95\% CI & OR 95\% CI & OR 95\% CI & OR 95\% CI \\
\hline PM10 & $1.00(0.92-1.10)$ & $0.96(0.86-1.08)$ & $1.15(1.03-1.28)$ & $1.01(0.88-1.14)$ & $1.33(0.93-1.91)$ \\
PM2.5-10 & $0.99(0.91-1.08)$ & $0.95(0.85-1.05)$ & $1.15(1.04-1.27)$ & $1.00(0.88-1.12)$ & $1.20(0.87-1.66)$ \\
PN0.1-1.0 & $1.02(0.92-1.11)$ & $0.98(0.88-1.10)$ & $1.13(1.01-1.26)$ & $1.02(0.90-1.15)$ & $1.48(1.02-2.13)$ \\
PN0.01-0.1 & $1.06(0.91-1.24)$ & $1.04(0.89-1.21)$ & $1.01(0.86-1.18)$ & $1.06(0.90-1.25)$ & $1.71(0.98-2.95)^{\dagger}$ \\
Res. PM10 & $0.96(0.86-1.08)$ & $1.07(0.95-1.22)$ & $1.05(0.93-1.18)$ & $0.98(0.86-1.12)$ & $1.24(0.76-2.00)$ \\
Black carbon & $1.01(0.92-1.09)$ & $0.94(0.84-1.05)$ & $1.16(1.04-1.29)$ & $0.98(0.87-1.10)$ & $1.25(0.88-1.77)$ \\
\hline
\end{tabular}

Odds ratios (OR) and $95 \%$ confidence intervals (CI) refer to an increase in the pollutant by one interquartile range. *: adjusted for day, day $^{2}$, weekend, minimum temperature (lag 1), relative humidity (lag 1), pollen (lag 1) and first-order autocorrelation. ${ }^{\dagger}: \mathrm{p}<0.05 ;$ $\mathrm{p}<0.10$. For definitions see footnote to table 1 .

and death in rats $[15,16]$. These effects were observed after 30 -min exposure to a concentration of $0.7-1.0 \times 10^{6} \mathrm{n} \cdot \mathrm{cm}^{-3}$ with median particle size of $0.026 \mu \mathrm{m}$. Studies using titanium dioxide have also indicated that particles in the ultrafine size range are more toxic than larger particles, at equivalent masses [14]. In epidemiological studies the number concentration of ultrafine particles has been found to be associated with decreased lung function and increased respiratory symptoms at least as strongly as the mass of coarse particles [11-13].

The increase in PM10 was observed to be associated with the increase in daily outpatient visits for asthma and for upper respiratory illness among adults in Anchorage, Alaska, USA, where the PM10 is primarily composed of earth crustal material and volcanic ash [8]. In a study in rural eastern Washington State, USA, where particulates are volcanic in origin, an association was found between PM10 and the number of emergency room visits for bronchitis and sinusitis [7]. PM10 was also associated with an increased risk for respiratory hospital admissions in Spokane, Washington, USA, where the major source of PM10 is windblown dust [9]. The present study is consistent with these studies in that resuspended PM10 may also have adverse health effects, although the observed effect was smaller and inconsistent for different lags.

Results from a study among asthmatic adults in Erfurt, in the former East Germany, suggest that the number concentration of ultrafine particles would be more strongly associated with PEF and symptoms than the mass of fine and coarse particles [12]. Among asthmatic children in Finland, however, daily variations in PM10 were associated with impaired pulmonary function at least as strongly as the number concentration of ultrafine particles [13]. In these studies in Germany and Finland, particulates were mainly from combustion processes. The present study implies that the number concentration of fine particles may affect PEF, though the results were variable by lag.

An alternative explanation for the mechanism of the health effects of particulates is that the chemical composition of the particles, especially soluble transition metals, attached to the surface of the particles, determines their health effects [32-35]. Particles with higher soluble transition metal content have been observed to induce a greater degree of lung injury in rats and greater toxicity in lung fibroblasts [34, 35]. In addition, airborne iron has been found to be associated with exacerbation of respiratory symptoms and with medication use for airway symptoms [33].
In previous epidemiological studies, particulate air pollution has usually been measured as PM10, PM2.5 or black smoke. However, the chemical composition and size distribution of particulates varies greatly from country to country, from season to season, and sometimes from day to day. Therefore, measuring only PM10 or PM2.5 will probably give an inaccurate estimate of the health effects of particulate air pollution. As air quality guidelines have previously been driven by epidemiological studies, future epidemiological studies should have a high-technology assessment of the size distribution and the chemical composition of the particulate air pollution to be able to determine which aspect of particulate air pollution is responsible for the health effects. This information can then be used to design efficient monitoring techniques and control strategies.

In conclusion, no consistent effect of particles was found as the associations varied by lag. Also, given the short duration of the present study, separating the effects of different types of particles was difficult. However, the present study demonstrated the highly variable size and number distribution and chemical composition of particulates in Finland, and underlines the importance of also measuring the size and chemical composition of particles to determine which types of particles are associated with health effects.

\footnotetext{
Acknowledgements. The authors thank E. Pärjälä (City of Kuopio) for collecting the air pollution data on gaseous pollutants.
}

\section{References}

1. Dockery DW, Pope CA III, Xu X, et al. An association between air pollution and mortality in six US cities. $N$ Engl J Med 1993; 329: 1753-1759.

2. Dockery DW, Pope CA III. Acute respiratory effects of particulate air pollution. Annu Rev Publ Health 1994; 15 : $107-132$.

3. Schwartz J. What are people dying of on high air pollution days? Environ Res 1994; 64: 26-35.

4. Burnett RT, Dales R, Krewski D, Vincent R, Dann T, Brook JR. Associations between ambient particulate sulfate and admissions to Ontario hospitals for cardiac and respiratory diseases. Am J Epidemiol 1995; 142: 15-22.

5. Johnson KG, Loftsgaarden DO, Gideon RA. The effects of Mount St. Helens volcanic ash on the pulmonary function of 120 elementary school children. Am Rev Respir Dis 1982; 126: 1066-1069. 
6. Buist AS, Vollmer WM, Johnson LR, Bernstein RS, McCamant LE. A four-year prospective study of the respiratory effects of volcanic ash from Mt. St. Helens. Am Rev Respir Dis 1986; 133: 526-534.

7. Hefflin BJ, Jalaludin B, McClure E, et al. Surveillance for dust storms and respiratory diseases in Washington State, 1991. Arch Environ Health 1994; 49: 170-174.

8. Gordian ME, Özkaynak H, Xue J, Morris SS, Spengler JD. Particulate air pollution and respiratory disease in Anchorage, Alaska. Environ Health Perspect 1996; 104: 290-297.

9. Schwartz J. Air pollution and hospital admissions for respiratory disease. Epidemiology 1996; 7: 20-28.

10. Seaton A, Macnee W, Donaldson K, Godden D. Particulate air pollution and acute health effects. Lancet 1995; 345: 176.

11. Vedal S, Petkau J, White R, Blair J. Are adverse health effects of ambient particle exposure related more to particle number or particle mass? Am J Respir Crit Care Med 1996; 153: A16.

12. Peters A, Wichmann HE, Tuch T, Heinrich J, Heyder J. Respiratory effects are associated with the number of ultrafine particles. Am J Respir Crit Care Med 1997; 155: 1376-1383.

13. Pekkanen J, Timonen KL, Ruuskanen J, Reponen A, Mirme A. Effects of ultrafine and fine particles in urban air on peak expiratory flow among children with asthmatic symptoms. Environ Res 1997; 74: 24-33.

14. Ferin J, Oberdörster G, Penney DP. Pulmonary retention of ultra-fine and fine particles in rats. Am J Respir Cell Mol Biol 1992; 6: 535-542.

15. Warheit DB, Seidel WC, Carakostas MC, Hartsky MA. Attenuation of perfluoropolymer fume pulmonary toxicity: effects of filters, combustion method and aerosol age. Exp Mol Pathol 1990; 52: 309-329.

16. Oberdörster G, Gelein RM, Ferin J, Weiss B. Association of particulate air pollution and acute mortality: involvement of ultrafine particles? Inhalation Toxicol 1995; 7: 111-124.

17. Hosiokangas J, Kikas Ü, Pekkanen J, Ruuskanen J, Tammet $\mathrm{H}$. Identifying and quantifying air pollution sources in Kuopio by receptor modeling. J Aerosol Sci 1995; 26: Suppl. 1, S423-424.

18. Timonen KL, Pekkanen J, Korppi M, Vahteristo M, Salonen RO. Prevalence and characteristics of children with chronic respiratory symptoms in eastern Finland. Eur Respir J 1995; 8: 1155-1160.

19. Roemer W, Hoek G, Brunekreef B, et al. Daily variations in air pollution and respiratory health in a multicentre study: the PEACE project. Eur Respir J 1998; 12: 13541361.

20. Remes ST, Korppi M, Remes K, Pekkanen J. Prevalence of asthma at school age: a clinical population-based study in eastern Finland. Acta Paediatr 1996; 85: 59-63.
21. Brunekreef B, ed. Effects of short-term changes in urban air pollution on the respiratory health of children with chronic respiratory symptoms. Study procedures for collaborative study funded by the Commission of the European Communities in the framework of the 'ENVI-

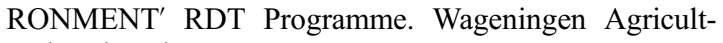
ural University, 1993.

22. Mirme A. Electric Aerosol Spectrometry. PhD Thesis, University of Tartu, 1994.

23. Marple VA, Rubow KL, Turner W, Spengler JD. Low flow rate sharp cut impactors of indoor air sampling: design and calibration. J Air Pollut Control Assoc 1987; 37 : 1303-1307.

24. Hansen ADA, Rosen H, Novakov T. The Aethalometeran instrument for real-time measurement of optical absorption by aerosol particles. Sci Total Environ 1984; 36: 191-196.

25. The Finnish Pollen Bulletin, Vol 20. Aerobiology Unit, University of Turku, 1995.

26. SAS Institute Inc. SAS/STAT® User's Guide, Version 6, Fourth Edition, Volume 2. Cary, NC, SAS Institute Inc., 1989; pp. 891-996.

27. SAS Institute Inc. SAS/STAT® Software: Changes and Enhancements through Release 6.11. Cary, NC, SAS Institute Inc., 1996; pp. 531-656.

28. The Finnish Pollen Bulletin, Vol 19 Suppl. Aerobiology Unit, , University of Turku, 1994.

29. Statistical Sciences. S-PLUS Guide to Statistical and Mathematical Analysis, Version 3.3. Seattle, StatSci, 1995; pp. 216-217.

30. SAS Institute Inc. SAS/STAT® User's guide, Version 6, Second Edition. Cary, NC, SAS Institute Inc., 1993; pp. $183-254$.

31. Littell RC, Milliken GA, Stroup WW, Wolfinger RD.

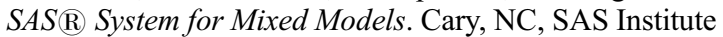
Inc., 1996; pp. 423-460.

32. Tepper JS, Lehmann JR, Winset DW, Costa DL, Ghio AJ. The role of surface complexed iron in the development of acute lung inflammation and airway hyperresponsiveness. Am J Respir Crit Care Med 1994; 149: A839.

33. Dusseldorp A, Kruize H, Brunekreef B, Hofschreuder P, de Meer G, van Oudvorst AB. Associations of PM10 and airborne iron with respiratory health of adults living near a steel factory. Am J Respir Crit Care Med 1995; 152: 1932-1939.

34. Osornio-Vargas AR, Alfaro-Moreno E, Rosas I, et al. The in vitro toxicity of ambient PM10 particles form the southern, central and northern regions of Mexico City to lung fibroblasts is related to transition metal content. Am J Respir Crit Care Med 1996; 153: A15.

35. Dreher K, Jaskot R, Richards J, et al. Acute pulmonary toxicity of size-fractionated ambient air particulate matter. Am J Respir Crit Care Med 1996; 153: A15. 